\title{
Dietary recommendations for patients with nonalcoholic fatty liver disease
}

\author{
Angelika Kargulewicz, Hanna Stankowiak-Kulpa, Marian Grzymisławski \\ Department of Internal Medicine, Metabolism and Dietetics, Poznan University of Medical Sciences, Poland
}

Prz Gastroenterol 2014; 9 (1): 18-23 DOI: $10.5114 / p g .2014 .40845$

Key words: nonalcoholic fatty liver disease, diet, dietary recommendations.

Address for correspondence: Angelika Kargulewicz, os. Orła Białego 77/10, 61-251 Poznan, Poland, phone: +48 668541699 , e-mail: angelikak610@gmail.com

\begin{abstract}
Changes to patients' lifestyle, especially a modified dietary approach, play a key role in the treatment of nonalcoholic fatty liver disease (NAFLD). A balanced, limiting and individually tailored nutritional scheme enables weight loss and an improvement in the clinical picture of NAFLD. According to nutritional recommendations for patients with NAFLD, carbohydrates should comprise $40-50 \%$ of total dietary energy. It is advisable to increase the amount of complex carbohydrates rich in dietary fibre. A major role in the aetiology of NAFLD is played by excessive intake of fructose, which is related to the rise in consumption of nonalcoholic beverages among subjects in developed countries. Fat intake should comprise $<30 \%$ of daily calories. It is essential to increase consumption of food products rich in mono- and polyunsaturated fatty acids. Ingestion of protein should constitute $15-20 \%$ of total energy.
\end{abstract}

\section{Introduction}

Lifestyle alteration, including dietary recommendations, plays an important role in the treatment of nonalcoholic fatty liver disease (NAFLD). A well composed, restrictive diet tailored to personal demands enables weight loss and improvement in the clinical picture of NAFLD [1]. The diet should be low in calories and rich in unsaturated fatty acids and natural antioxidants (vitamins $A$ and $C$ ). According to the nutritional recommendations for patients with NAFLD, carbohydrates should comprise $40-50 \%$ of total dietary energy. It is advisable to increase the amount of complex carbohydrates rich in dietary fibre. A substantial role in the aetiology of NAFLD is played by excessive intake of fructose caused by the high consumption of nonalcoholic beverages among subjects in developed countries. Fructose intake causes the development of fatty liver through stimulation of de novo lipogenesis. Ingestion of simple carbohydrates should not exceed $10 \%$ of total energy intake.

Fat intake should comprise $<30 \%$ of daily calory intake. It is essential to increase consumption of food products rich in mono- and polyunsaturated fatty acids. Mono- and polyunsaturated fatty acids lessen inflammation and contribute to improvement of the clinical picture of NAFLD. Ingestion of protein should constitute $15-20 \%$ of total energy [1-3]. Moderate intake of protein is optimum for patients with NAFLD, due to the alleviation of insulin resistance.

\section{Weight loss}

Weight loss is advisable for overweight (body mass index (BMI): $25-30 \mathrm{~kg} / \mathrm{m}^{2}$ ) and obese (BMI > $30 \mathrm{~kg} / \mathrm{m}^{2}$ ) patients. It should not exceed $1 \mathrm{~kg}$ per week. Rapid and uncontrolled weight loss can be detrimental for patients and may even worsen clinical symptoms of NAFLD $[4,5]$. It is recommended to avoid very low calorie diets (388 kcal/day). They can cause the activation of overall inflammation and a rise in serum bilirubin levels $[3,6]$.

\section{The impact of nutrients on the development of nonalcoholic fatty liver disease}

The impact of nutrients on the development of NAFLD can be observed from as early as foetal life. A study conducted on rats revealed that an unbalanced diet during pregnancy and lactation contributes to the development of NAFLD in offspring. Maternal diet during pregnancy rich in energy, fat, sugar and salt increases 
the risk of NAFLD development and stimulates oxidative stress in offspring. Metabolic disturbances fixed during pregnancy and lactation cannot be turned back after introduction of a sensible diet in weaning time. A crucial role in the prevention of metabolic disturbances (e.g. NAFLD) in offspring is played by nutritional education of pregnant women [7].

\section{Saturated fatty acids}

A study conducted on rats revealed that a diet rich in saturated fatty acids (SFAs) stimulates oxidative stress in mitochondria and contributes to the damage of hepatocytes [8]. Gentile et al. showed in a study conducted on Wistar rats that a high ratio of saturated to unsaturated fatty acids in subjects with NAFLD increases the susceptibility of hepatocytes to endotoxin action and induces progression of simple steatosis towards nonalcoholic steatohepatitis (NASH) [9]. Exposure of hepatocytes to stearic and palmitic acids causes their apoptosis through activation of caspase 3 and stimulation of DNA fragmentation. Dietary recalls collected from patients with NASH revealed that they consume more saturated fatty acids ( $14 \%$ of total energy intake) than control subjects (10\% of total energy intake). Intake of saturated fatty acids more than $10 \%$ of total energy may stimulate insulin resistance. Intake of saturated fatty acids less than $10 \%$ of total energy diminishes serum LDL and triglyceride levels. Restriction of SFA intake to less than $7 \%$ of total energy does not lead to alleged improvements in lipid profile and may even be detrimental for the patient. Recommended daily intake of SFAs should be more than $7 \%$ but less than $10 \%$ of total energy intake [8].

\section{Trans fatty acids}

Trans fatty acids occur naturally in dairy products. They are synthesised by bacterial microbiota in the gastrointestinal tract of ruminants. Trans fatty acids can also be made commercially during the production of margarine. They are formed during the hydrogenation of plant oils. Conjugated linoleic acid (isomer cis-9 trans-11) contained in dairy products does not negatively influence the human organism. On the other hand, trans fatty acids contained in hydrogenated margarine exhibit the opposite effect. The intake of conjugated linoleic acid (isomer trans-10 cis-12) in the form of hydrogenated margarine promotes proinflammatory action, intensifies endothelium dysfunction and predisposes towards lipid profile disturbances. Dietary recommendations in NAFLD suggest avoidance of the trans fatty acids contained in highly processed food products [8].

\section{Monounsaturated fatty acids}

Ingestion of monounsaturated fatty acids (MUFAs) lowers the risk of development of cardiovascular disease and contributes to the improvement of lipid profile. Food products rich in MUFAs include olive oil, rapeseed oil, sunflower, soy, nuts and avocado. Replacement of dietary saturated fatty acids by monounsaturated fatty acids in a diet causes amelioration of serum glucose level and blood pressure. A diet rich in MUFAs increases the level of HDL-cholesterol and decreases the level of total cholesterol in subjects with diabetes [8]. Monounsaturated fatty acids inhibitoxidation of LDL-cholesterol and decrease the serum level of triglycerides by activation of peroxisome proliferator-activated receptor $\alpha$ (PPAR $\alpha)$ [10]. A study conducted on humans with NASH confirmed the beneficial effect of a MUFA-rich diet on oxidative stress [11, 12]. A food product notably rich in MUFAs is olive oil (74 $\mathrm{g}$ of MUFAs per $100 \mathrm{~g})$. Numerous clinical trials confirm the favourable effect of olive oil on the prevention and treatment of metabolic syndrome [10]. Unrefined, extra virgin olive oil contains a number of bioactive constituents with antioxidative properties (Table I). A diet enriched with olive oil ameliorates endothelial function and inhibits TNF- $\alpha$ production [10].

A diet rich in MUFAs (40\% of total energy intake) leads to a decrease in very low density lipoproteins (VLDL) levels to a higher degree than a diet rich in carbohydrates (concomitant fat intake: $28 \%$ of total calories) does. Additionally, diet rich in MUFAs is accepted organoleptically by patients [8].

Table I. The influence of olive oil on fatty liver [10]

\begin{tabular}{|c|c|}
\hline Constituent & Mechanism of action \\
\hline $\begin{array}{l}\text { Oleic acid } \\
\text { Phenols }\end{array}$ & $\begin{array}{l}\text { Anti-inflammatory and } \\
\text { immunomodulatory effects }\end{array}$ \\
\hline $\begin{array}{l}\text { Phenols: } \\
\text { Hydroxytyrosol } \\
\text { Oleuropein } \\
\text { Caffeic acid } \\
\text { O-coumaric acid } \\
\text { Vanillic acid } \\
\text { 3,4-Dihydroxyphenyl ethanol }\end{array}$ & $\begin{array}{l}\text { Decreased lipid oxidation } \\
\text { Decreased DNA damage }\end{array}$ \\
\hline Oleic acid & $\begin{array}{l}\text { Decreased synthesis } \\
\text { of arachidonic acid }\end{array}$ \\
\hline Hydroxytyrosol & Inhibits lipo-oxygense \\
\hline Squalene & $\begin{array}{l}\text { Inhibits HMG-CoA reductase } \\
\text { Inhibits RAS activation }\end{array}$ \\
\hline $\begin{array}{l}\text { Oleic acid } \\
\text { Lignans }\end{array}$ & $\begin{array}{l}\text { Decreased membrane } \\
\text { permeability and oxidation }\end{array}$ \\
\hline Protocatechuic acid & $\begin{array}{l}\text { Decreased synthesis } \\
\text { of arachidonic acid }\end{array}$ \\
\hline
\end{tabular}




\section{Polyunsaturated fatty acids}

Polyunsaturated fatty acids, including docosahexaenoic acid (DHA) and eicosapentaenoicacid (EPA), positively affect the course of NAFLD. Food products rich in EPA and DHA include sea fish, green leafy vegetables, rapeseed oil and flax seeds. Consumption of fish oil decreases de novo lipogenesis, increases glucose uptake in fat tissue and reduces visceral fat [8, 13]. The intake of omega-3 fatty acids inhibits activation of Kupffer cells and output of proinflammatory cytokines. Spadaro revealed in his research that omega-3 supplementation (dose: $2 \mathrm{~g} /$ day for 6 months) ameliorates the course of NAFLD by decreasing the level of steatosis [14, 15]. Experimental studies on animals pointed out that fish oil supplements with a high content of EPA and DHA exhibit positive effect in subjects with insulin resistance (Table II) [8].

The DHA and EPA deficiency can contribute to the development of NAFLD, which was confirmed in an experimental study on animals [13]. Studies conducted on humans have revealed that decreased availability of omega-3 fatty acids intensifies fatty acids synthesis and lipid accumulation within hepatocytes [11]. The recommended omega- 3 to omega- 6 ratio should be $1: 1$ to $1: 4$, which is a crucial issue. The source of omega- 6 fatty acids are: cereals, eggs and animal fat [2]. Patients with NASH exhibit a high consumption of omega- 6 fatty acids and an abnormal omega-3: omega- 6 ratio [16].

Replacement of omega- 6 fatty acids by $\alpha$-linolenic fatty acid (omega-3) increased insulin sensitivity and lowered serum cholesterol levels in a high-fructose experimental model. The consumption $6 \%$ of total calories as omega- 6 fatty acids and $1 \%$ as omega- 3 fatty acids reduces the risk of cardiovascular disease development [8].

The intake of omega- 3 fatty acids significantly ameliorates lipid profile and reduces inflammatory response, steatosis and hepatocytes damage in NAFLD patients [8].

Table II. The effect of fish oil on subjects with insulin resistance [8]

Decrease in triglycerides, free fatty acids, glucose and insulin serum level

Alleviation of peripheral insulin resistance

Decrease in very low density lipoprotein (VLDL) secretion and inhibition of hepatic lipogenesis

Increase in utilisation and storage of glucose in skeletal muscles

Reduction of the adipocyte size and visceral fat content

Activation of insulin-dependent glucose transporter

in the adipose tissue

\section{Simple carbohydrates}

Worldwide changes in dietary habits have caused contemporary populations to consume more and more highly processed food products rich in simple carbohydrates [17]. A number of studies have shown a significant relationship between high consumption of soft drinks and risk for obesity and diabetes development, especially in children and adolescents [18]. Highly processed food is often rich in fructose and saccharose. These simple carbohydrates are very often used in the production of food. Fructose is used as a sweetener in the production of juices, jellies and jams. Consumption of soft drinks and juices rich in high-fructose corn syrup (HFCS) has increased form $3.9 \%$ of total calories in 1977 to $9.2 \%$ of total calories in 2001. In addition, soft drinks contain considerably high amounts of aspartame and caramel colorant. Caramel colorant is a high source of advanced glycation end products (AGEs) which exhibit pro-inflammatory properties. Aspartame is a peptide ester which is 160 times sweeter than saccharose. The intake of processed food high in aspartame causes weight gain and increases the risk for diabetes type 2 development [17]. Consumption of soft drinks rich in fructose contributes to insulin resistance and furthermore stimulates NAFLD development. Fructose intensifies lipogenesis and triglycerides synthesis. Studies conducted on ducks and rats revealed that a high-fructose diet causes liver steatosis [17, 19].

Soft drinks with a high fructose content include Soda, Soda Pop, Coca-cola and Tonic [4]. In a study conducted on adult men it was proven that a normo-caloric diet with a $3 \mathrm{~g}$ of fructose per $\mathrm{kg}$ of body mass per day (about $4 \mathrm{I}$ of high-fructose soft drink) contributes to increased accumulation of lipids within hepatocytes, decreased insulin sensitivity and increased triglycerides serum level [20]. Consumption of more than one serving of a high-fructose soft drink (about $360 \mathrm{ml}$ ) per day significantly increases the risk of metabolic syndrome development. That risk is not observed in subjects who do not consume any high-fructose soft drinks [17, 18].

\section{Oligofructose- prebiotics used in nutritional therapy of nonalcoholic fatty liver disease}

Oligofructose is an indigestible fructose oligomer that is found in plant food. Food products rich in oligofructose include chicory, artichoke, leek, asparagus and garlic. Clinical trials conducted on humans showed that oligofructose ingestion causes a reduction in triglycerides and glucose serum levels. Oligofructose supplementation in overweight and obese patients contributes to a decrease in postprandial glucose levels and it beneficially 
influences glycaemia control [21]. A diet rich in oligofructose stimulates satiety through bacterial fermentation and the increase in free fatty acid concentrations in the large intestine [22]. In a study conducted on rats it was proven that oligofructose supplementation causes a reduction in the amount of consumed food and increases serum glucagon-like peptide 1 concentration (GLP-1). Another trial revealed that GLP-1 supplementation for 6 months favourably regulates glycaemia, contributes to weight loss and reduces the amount of ingested food. Oligofructose supplementation reduces the risk of insulin resistance, diabetes and obesity development in animals fed a high-fat diet (fat content: $72 \%$ of total calories) [22]. Additionally, oligofructose consumption attenuates the inflammation within hepatocytes [21]. The influence of oligofructose is allegedly related to the action of GLP-1 and its concentration in brain cells and serum [22]. Oligofructose intake also causes a decrease in a ghrelin concentration. Ghrelin is an orexigenic hormone which stimulates food ingestion [21].

\section{Protein content - diet in nonalcoholic fatty liver disease}

Clinical trials show that protein intake has a beneficial impact on the course of NAFLD. Protein deficiency, as well as malnutrition, can lead to NASH development [12]. Studies conducted on rodents revealed that a high-protein and low-carbohydrate diet ameliorates carbohydrate metabolism and decreases liver steatosis by inhibition of de novo lipogenesis. Dietary protein has a beneficial effect on carbohydrate metabolism and decreases insulin output. Protein intake is essential for the regeneration of hepatocytes and supplies crucial amino acids that prevent excessive fat accumulation within hepatocytes [12]. A high-protein diet (40\% of total calories as protein) decreases body fat content and reduces the risk of cardiovascular disease development more than a diet which supplies $15 \%$ of total calories as protein does [23]. On the other hand, excessive protein intake can cause glomerular sclerosis, renovascular hypertension and kidney dysfunction in patients with kidney failure [8]. Arciero in his research proved that a moderate protein intake ( $25 \%$ of total calories as protein) is as efficient in reducing body fat content as a high-protein diet is. It is said that a moderate protein intake is optimum for patients with NAFLD, due to the positive impact on weight loss and insulin sensitivity. Protein intake as $25 \%$ of total calories in patients with NAFLD does not cause side effects [23].

\section{Monosodium glutamate}

Recently, the intake of food products with added monosodium glutamate (MSG) has significantly in- creased. It is estimated that the consumption of MSG in Europe amounts $30 \mathrm{mg} / \mathrm{kg}$ of body weight per day. Acceptable daily intake (ADI) is $0-120 \mathrm{mg} / \mathrm{kg}$ of body weight. Monosodium glutamate is a food additive used to enhance flavour in any number of foods. It is especially prevalent in processed, frozen and canned food. Monosodium glutamate is commonly used in Asian cuisine $[24,25]$.

Current clinical studies show that MSG consumption increases the risk of fatty liver development and enhances inflammation as well as dysplasia within hepatocytes. Monosodium glutamate intake contributes to the rise in free fatty acids and triglyceride serum concentrations. Exposition to MSG causes deterioration in fatty acid $\beta$-oxidation, bile acid synthesis and lipid storage [26].

\section{Nutritional recommendations for patients with nonalcoholic fatty liver disease}

A diet recommended for patients with NAFLD should be low in calories and rich in dietary fibre and monounsaturated fatty acids (Table III) [1, 2].

\section{Food product choice}

Food products recommended for patients with NAFLD should be low in saturated fatty acids $[6,27,28]$. It is advisable to eliminate soft drinks rich in a high-fructose corn syrup [29]. Meat should be low in fat. Recommended meats are white meat and occasionally red meat. Food should be boiled, steamed or baked without added

Table III. Dietary recommendations for subjects with obesity and nonalcoholic fatty liver disease [1-3]

Energy content:
- $1000-1200 \mathrm{kcal}$ per day for obese women*
- $1200-1600 \mathrm{kcal}$ per day for obese men*
*Energy requirement should be tailored to personal needs. Physical
activity, height and weight should be considered.
Nutrients in diet:
- Carbohydrates should comprise $40-50 \%$ of total calories
- Fat should comprise $\leq 30 \%$ of total calories (saturated fatty
acids > $7 \%$ and < $10 \%$ of total calories)
- Protein should comprise about $20 \%$ of total calories
Energy deficit should be between 500 and 1000 kcal per day.
Above mentioned deficit enables weight loss $0.5-1$ kg per week
Meal intake:
- It is recommended to consume $4-5$ meals per day; breaks
between meals should not exceed $2-3 \mathrm{~h}$
- Last meal should be consumed at least $3 \mathrm{~h}$ before sleeping
- Meals should be consumed slowly
- It is important to finish eating when the patient does not feel
satiety; signal of satiety is felt usually 15 min after the end
of consumption


fat. Frying should be avoided. Fried food is difficult to digest and the calory content is considerably higher than that of steamed or boiled food. Using cream or roux is contraindicated. Fast food should be excluded [6].

Walnuts are recommended for NAFLD patients. They are rich in $\alpha$-linolenic fatty acid. Consumption of $30 \mathrm{~g}$ of walnuts per day causes a decrease in total cholesterol and LDL levels [8]. The diet should be rich in fruit and vegetables, which are good sources of antioxidative vitamins ( $\beta$-carotene, vitamins $C$ and $E$ ). Good sources of vitamin C include blackcurrants $(182.6 \mathrm{mg} / 100 \mathrm{~g}$ of product), parsley leaves (177 mg/100 g), red peppers (144 $\mathrm{mg} / 100 \mathrm{~g}$ ) and horseradish $(114 \mathrm{mg} / 100 \mathrm{~g})$. Food products rich in $\beta$-carotene are: carrot $(9938 \mu \mathrm{g} / 100 \mathrm{~g})$, dried apricot $(7842 \mu \mathrm{g} / 100 \mathrm{~g})$, parsley leaves $(5410 \mu \mathrm{g} / 100 \mathrm{~g})$ and onion leaves $(3400 \mu \mathrm{g} / 100 \mathrm{~g})$. Good sources of vitamin E include plant oils, especially sunflower oil $(46.71 \mathrm{mg} /$ $100 \mathrm{~g})$, fortified margarines $(27.72 \mathrm{mg} / 100 \mathrm{~g})$ and wheat germ $(15.10 \mathrm{mg} / 100 \mathrm{~g})$ [30].

A recommended fruit in the diet of patients with NAFLD is aroniae juice and preserves. Aroniae is characterised by a high antioxidative potential in comparison to other fruits. Aroniae contains polyphenols and especially high amounts of proanthocyanins and anthocyanins. Aroniae juice inhibits LDL oxidation and minimises the detrimental effects of fructose intake. Aroniae extract counteracts fructose-induced hypertriglyceridaemia and decreases body weight [31, 32].

\section{References}

1. York LW, Puthalapattu S, Wu GY. Nonalcocholic fatty liver dis ease and low-carbohydrate diets. Annu Rev Nutr 2009; 29: 365-79.

2. Bellentani S, Grave RD, Suppini A. Behavior therapy for nonalcoholic fatty liver disease: the need for a multidisciplinary approach. Hepatology 2008; 47: 746-54.

3. Adams LA, Angulo P. Treatment of non-alcoholic fatty liver disease. Postgrad Med J 2006; 82: 315-22.

4. Dudzik D, Knaś M, Borzym-Kluczyk M, et al. Nonalcoholic steatohepatitis (NASH) - pathogenesis, diagnosis, treatment [Polish]. Med Sci Hepatol 2008; 8: 48-58.

5. Raszeja-Wyszomirska J, Ławniczak M, Marlicz W, et al. Nonalcoholic fatty liver disease (NAFLD) - new insight [Polish]. Pol Merkuriusz Lek 2008; 24: 568-71.

6. Jarosz M, Respondek W. Dietary recommendations and lifestyle [Polish]. In: Nonalcoholic fatty liver disease. Jarosz M (ed.) PZWL, Warsaw 2010; 45-56.

7. Bayol SA, Simbi BH, Fowkes RC. A maternal "junk food” diet in pregnancy and lactation promotes nonalcoholic fatty liver disease in rat offspring. Endocrinology 2010; 151: 1451-61.

8. Zivkovic AM, German JB, Sanyal AJ. Comparative review of diets for the metabolic syndrome: implications for nonalcoholic fatty liver disease. Am J Clin Nutr 2007; 86: 285-300.
9. Gentile CL, Pagliassotti MJ. The role of fatty acids in the development and progression oh nonalcoholic fatty liver disease. J Nutr Biochem 2008; 19: 567-76.

10. Assy N, Nassar F, Nasser G, et al. Olive oil consumption and non-alcoholic fatty liver disease. World J Gastroenterology 2009; 15: 1809-15.

11. Vos MB, McClein CJ. Nutrition and nonalcoholic fatty liver disease in children. Curr Gastroenterol Rep 2008; 10: 308-15.

12. Leclercq IA, Horsmans Y. Nonalcoholic fatty liver disease: the potential role of nutrition management. Curr Opin Clin Nutr Metab Care 2008; 11: 766-73.

13. Byrne C, Olufadi R, Bruce KD. Metabolic disturbances in non-alcoholic fatty liver disease. Clin Sci 2009; 116: 539-64.

14. Spadaro L, Magliocco O, Spampinato D, et al. Effects of $n-3$ polyunsaturated fatty acids in subjects with nonalcoholic fatty liver disease. Dig Liv Dis 2008; 40: 194-9.

15. Pickova J. Importance of knowledge on lipid composition of foods to support development towards consumption of higher levels of n-3 fatty acids via freshwater fish. Physiol Res 2009; 58: 39-45.

16. Moschen AR, Tilg $H$. Nutrition in pathophysiology and treatment of nonalcoholic fatty liver disease. Curr Opin Clin Nutr Metab Care 2008; 11: 620-5

17. Nseir W, Nassar F, Assy N. Soft drinks consumption and nonalcoholic fatty liver disease. World J Gastroenterol 2010; 16 : 2579-88.

18. Dhingra R, Sullivan L, Jacques PF. Soft drink consumption and risk of developing cardiometabolic risk factors and the metabolic syndrome in middle-aged adults in community. Circulation 2007; 116: 480-8.

19. Abid A, Taha O, Nseir W. Soft drink consumption is associated with fatty liver disease independent of metabolic syndrome. J Hepatol 2009; 51: 918-24.

20. Le KA, Bortolotti M. Role of dietary carbohydrates and macronutrients in the pathogenesis of nonalcoholic fatty liver disease. Curr Opin Clin Nutr Metab Care 2008; 11: 477-82.

21. Parnell JA, Reimer RA. Weight loss during oligofructose supplementation is associated with decreased ghrelin and increased peptide $Y Y$ in overweight and obese adults. Am J Clin Nutr 2009; 89: 1751-9.

22. Cani PD, Knauf C, Iglesias MA. Improvement of glucose tolerance and hepatic insulin sensitivity by oligofructose requires a functional glucagon-like peptide 1 receptor. Diabetes 2006; 55: 1484-90.

23. Arciero PJ, Gentile CL, Pressman R, et al. Moderate protein intake improves total and regional body composition and insulin sensitivity in overweight adults. Metabolism 2008; 57 : 757-65.

24. Krishna VN, Karthika D, Surya DM, et al. Analysis of monosodium l-glutamate in food products by high-performance thin layer chromatography. J Young Pharm 2010; 2: 297-300.

25. Gertig H, Duda G. Allowed substances added to food products [Polish]. In: Law in a food industry. Grtig H, Duda G (eds.). PZWL, Warsaw 2004; 209-10.

26. Collison KS, Maqbool Z, Inglis AL, et al. Effect of dietary monosodium glutamate on trans fat-induced nonalcoholic fatty liver disease. J Lipid Res 2009; 50: 1521-37. 
27. Nimatha MW, Day CP. Non-alcoholic fatty liver disease: the mist gradually clears. J Hepatol 2008; 48: 104-12.

28. Ciborowska H. Low-calorie diet [Polish]. In: Dietetics. Nutritional therapy for healthy and ill patients. Ciborowska $\mathrm{H}$, Rudnicka A (eds.). PZWL, Warsaw 2007; 280-9.

29. Loria P, Adinolfi LE, Bellentani S, et al. Practice guidelines for the diagnosis and management of nonalcoholic fatty liver disease. Dig Liv Dis 2010; 42: 272-82.

30. Ciborowska H, Rudnicka A. Nutrition of human with elements of biochemistry [Polish]. In: Dietetics. Nutritional therapy for healthy and ill patients. Ciborowska H, Rudnicka A (eds.). PZWL, Warsaw 2007; 280-9.

31. Fołta M, Bartoń H, Paśko P, et al. Oxidative stress and metabolic disturbances in a high-fructose rat model [Polish]. Bromat Chem Toksykol 2009; 3: 842-7.

32. Raszeja-Wyszomirska J, Safranow K, Szynkowska A. Secretory phospholipase A2 activity is linked to hipercholesterolemia and gender in non-alcoholic fatty liver disease individuals. Prz Gastroenterol 2013; 8: 172-5.

Received: $\quad 11.11 .2011$

Accepted: 17.03 .2012 